\title{
Liposomal paclitaxel induces fewer hematopoietic and cardiovascular complications than bioequivalent doses of Taxol
}

\author{
SHIH-TING HUANG ${ }^{1,2}$, YI-PING WANG ${ }^{2-4}$, YEN-HUI CHEN $^{5}$, CHIN-TARNG LIN $^{6}$, \\ WEN-SHAN LI ${ }^{1,7}$ and HAN-CHUNG WU ${ }^{1,2}$ \\ ${ }^{1}$ Graduate Institute of Life Sciences, National Defense Medical Center, Taipei 114; \\ ${ }^{2}$ Institute of Cellular and Organismic Biology, Academia Sinica, Taipei 115; \\ ${ }^{3}$ Graduate Institute of Clinical Dentistry, School of Dentistry, National Taiwan University; \\ ${ }^{4}$ Department of Dentistry, National Taiwan University Hospital, Taipei 100; \\ ${ }^{5}$ Institute of Biomedical Sciences, Academia Sinica, Taipei 115; \\ ${ }^{6}$ Department of Pathology, College of Medicine, National Taiwan University, \\ Taipei 100; ${ }^{7}$ Institute of Chemistry, Academia Sinica, Taipei 115, Taiwan, R.O.C.
}

Received February 28, 2018; Accepted June 6, 2018

DOI: $10.3892 /$ ijo.2018.4449

\begin{abstract}
Paclitaxel (PTX) exhibits potent antineoplastic activity against various human malignancies; however, clinical application must overcome the inherent hydrophobicity of this molecule. The commercialized Taxol formulation utilizes Cremophor EL (CrEL)/ethanol as a solvent to stabilize and dispense PTX in an aqueous solution. However, adverse CrEL-induced hypersensitivity reactions have been reported in $\sim 30 \%$ of recipients, and $40 \%$ of patients receiving premedication may also experience this adverse effect. Therefore, the development of a CrEL-free delivery system is crucial, in order to fully exploit the therapeutic efficacy of PTX. In the present study, a novel liposomal PTX (lipo-PTX) formulation was optimized with regards to encapsulation rate and long-term stability, arriving at a molar constituent ratio of soybean phosp hatidylcholine:cholesterol:N-(carbonyl-methoxy-poly-ethylene glycol 2000)-1,2-distearoyl-sn-glycero-3-phosphoethanolamine, sodium salt:PTX at 95:2:1:2. Comparable doses of lipo-PTX and Taxol were bioequivalent in terms of therapeutic efficacy in xenograft tumor models. However, the systemic side effects, including hematopoietic toxicity, acute hypersensitivity reactions and cardiac irregularities, were significantly
\end{abstract}

Correspondence to: Dr Han-Chung Wu, Institute of Cellular and Organismic Biology, Academia Sinica, 128 Academia Road, Section 2, Nankang, Taipei 115, Taiwan, R.O.C.

E-mail: hcw0928@gate.sinica.edu.tw

Dr Wen-Shan Li, Institute of Chemistry, Academia Sinica, 128 Academia Road, Section 2, Nankang, Taipei 115, Taiwan, R.O.C. E-mail: wenshan@gate.sinica.edu.tw

Key words: Taxol, Abraxane, paclitaxel, liposome, drug delivery system, cardiotoxicity reduced in lipo-PTX-treated mice compared with those infused with reference formulations of PTX. In conclusion, the present study reported that lipo-PTX exhibited a higher therapeutic index than clinical PTX formulations.

\section{Introduction}

Paclitaxel (PTX) is a natural-product anticancer drug that is isolated from the bark of Taxus brevifolia. This drug has generated much interest due to its broad-spectrum antineoplastic activity, which is mediated by preventing microtubule depolymerization and subsequently stimulating M-phase cell cycle arrest and apoptosis $(1,2)$. Furthermore, it has been reported that the chemotherapeutic use of PTX can induce generation of reactive oxygen species, increase production of hydrogen peroxide and stimulate the immune system to combat tumors (3-5). To overcome the extremely poor water solubility of PTX, the pharmaceutical company Bristol-Myers Squibb developed Taxol, which consists of PTX micelles in a solvent of 50:50 (v/v) Cremophor EL (CrEL) and dehydrated ethanol (6). At present, Taxol is broadly used as in antineoplastic regimen for the treatment of numerous types of cancer, including ovarian, breast, lung, head-andneck, prostate and pancreatic cancer, and acquired immune deficiency syndrome-related Kaposi's sarcoma $(1,7)$. However, CrEL is known to induce acute systemic side effects within 10 min of initiation of drug infusion, including anaphylactic hypersensitivity reactions (HSRs), hypotension, neutropenia, cardiotoxicity, neuropathy and hyperlipidemia (8-10). Consequently, corticosteroids and antihistamines are sometimes used as premedications. As an additional measure, Taxol infusion rate may be slowed to reduce the intensity and incidence of CrEL-associated toxicity. However, $\sim 40 \%$ of patients still suffer from HSRs, even after premedication (11). Alongside CrEL-mediated toxicity, Taxol-based delivery 
produces undesirable nonlinear pharmacokinetic behaviors associated with the disposition and metabolism of PTX $(12,13)$. Therefore, a CrEL-free delivery system for PTX is urgently required to mitigate the side effects and improve the pharmacokinetics.

Recently, various PTX delivery systems have been investigatedanddevelopedtoreduceCrEL-induced toxicitiesassociated with Taxol. Emulsions, micelles, polymers, nanocrystals, cyclodextrins, hydrogels, liposomes and water-soluble prodrugs have all been explored $(14,15)$. Among these novel formulations, the only one to successfully gain Food and Drug Administration (FDA) approval and commercialization is nanoparticle albumin-bound PTX, Abraxane, which is also called nab-PTX (Celgene Corporation, Summit, NJ, USA) $(16,17)$. Compared with Taxol, Abraxane significantly increases the maximum tolerated dosage (300 vs. $\left.175 \mathrm{mg} / \mathrm{m}^{2}\right)$, overall response rate $(33$ vs. $19 \%$ ) and time to progression (23.0 vs. 16.9 weeks) (18). In addition, numerous Taxol-associated adverse effects, including HSR, grade 3/4 neutropenia, hypotension and bradycardia, are alleviated in patients receiving Abraxane. However, electrocardiogram (ECG) irregularities are not rectified by Abraxane, and up to $60 \%$ of patients receiving Abraxane treatment exhibit abnormal ECG readings (19). Furthermore, the incidence of myocardial infarction is $44 \%$ in the first month following Abraxane treatment, which is decreased to $33 \%$ in the first half-year and $22 \%$ in the second half-year post-therapy (20). Although HSRs associated with Abraxane are significantly less frequent than with Taxol, myocardial toxicity remains a major issue (18-20). Therefore, a novel CrEL-free delivery system for PTX with reduced cardiac toxicity and a similar therapeutic profile to Taxol is urgently required to satisfy the clinical need.

For several drugs, liposomal formulations offer unsurpassed advantages, including the ability to carry a hydrophobic payload, ease of synthesis, favorable manufacturing control and excellent biocompatibility. Liposomal PTX has previously been reported to reduce CrEL-associated side effects at the expense of poor stability (21-25). The present study introduced a novel liposomal PTX (lipo-PTX) preparation that is composed of PTX/lipid at a molar ratio of $2 \%$, with a PTX concentration of $2 \mathrm{mg} / \mathrm{ml}$ in PBS. This lipo-PTX formulation exhibited long-term stability when stored at $4^{\circ} \mathrm{C}$, and possessed a similar therapeutic efficacy to Taxol in various cancer models. Notably, this novel formulation exhibited major reductions in CrEL-associated systemic side effects.

\section{Materials and methods}

Materials. Soybean phosphatidylcholine (SPC; Lipoid S100) and N-(carbonyl-methoxy-poly-ethylene glycol 2000)-1,2-distearoyl-sn-glycero-3-phosphoethanolamine, sodium salt (mPEG-DSPE; Lipoid PE 18:0/18:0PEG2000) were purchased from Lipoid GmbH (Ludwigshafen, Germany). Cholesterol (C8667) and MTT (M5655) were obtained from Sigma-Aldrich (Merck KGaA, Darmstadt, Germany). PTX (SPF2039) was provided by ScinoPharm Taiwan, Ltd. (Tainan, Taiwan). Oregon Green ${ }^{\circledR}$ 488-conjugated PTX (OG488-PTX; P22310) was purchased from Thermo Fisher Scientific, Inc. (Waltham, MA USA). Lissamine rhodamine B-conjugated 1,2-dipalmitoyl-sn-glycero-3-phosphoethanolamine-N-(lissamine rhodamine B sulfonyl) (ammonium salt) (Rh-DPPE; 810158P) was supplied by Avanti Polar Lipids (Alabaster, AL, USA). Taxol was obtained from Bristol-Myers Squibb Company (New York, NY, USA) and Abraxane was provided by Celgene Corporation. Roswell Park Memorial Institute (RPMI)-1640 medium (31800-022), Dulbecco's modified Eagle's medium (DMEM; 12100-061), DMEM/Nutrient Mixture F-12 (DMEM/F12; 12400-024), fetal bovine serum (FBS; 10437028), penicillin-streptomycin solution (P/S; 15140-122) and 2.5\% (w/v) trypsin solution (15090-046) were purchased from Gibco (Thermo Fisher Scientific, Inc.)

Animals. Non-obese diabetic/severe combined immunodeficiency (NOD/SCID, NOD.CB17-Prkdc ${ }^{\text {scid }} / \mathrm{J}$ ) mice (female; age, 4-6 weeks; weight, 18-22 g) were obtained from National Laboratory Animal Center (Taipei, Taiwan), and outbred ICR (Bltw:CD1) mice (female; age, 5-8 weeks; weight, 25-30 g) were supplied by BioLASCO Taiwan Co., Ltd. (Taipei, Taiwan). Mice were maintained in a specific pathogen-free animal room, with ad libitum access to food and water intake, at $18-23^{\circ} \mathrm{C}, 40-60 \%$ humidity under a 12 -h light/dark cycle at the Institute of Cellular and Organismic Biology, Academia Sinica (Taipei, Taiwan). All animal care and experimental procedures were conducted in accordance with the principles in the Guide for the Care and Use of Laboratory Animals (26), and the present study was approved by the Institutional Animal Care and Use Committee of Academia Sinica.

Preparation of PTX-loaded liposomes. PTX-loaded liposomes were prepared according to the thin film hydration method described previously (27-30). Briefly, SPC, cholesterol, mPEG-DSPE and PTX were dissolved in chloroform (C2432; Sigma-Aldrich; Merck KGaA) at a molar ratio of 95:2:1:2 in a round-bottom flask. A dry lipid film was formed on the bottom of the flask after the organic solvent was removed by rotary evaporation at $40^{\circ} \mathrm{C}$, and the film was hydrated in PBS ( $\mathrm{pH} \mathrm{7.4)}$ at $45^{\circ} \mathrm{C}$. The lipid suspension was downsized through 10 freeze-thaw cycles, and a LIPEX ${ }^{\circledR}$ Extruder (TRANSFERRA Nanosciences Inc., Burnaby, BC, Canada) with $0.2 \mu \mathrm{m}$ polycarbonate filter membranes (WHA110406; Sigma-Aldrich; Merck KGaA) was used to finally obtain homogeneous unilamellar liposomes. To evaluate the concentration and encapsulation efficiency of PTX, the encapsulated drug was extracted using $40 \%$ acetonitrile in fresh distilled-deionized water, and $40 \mu \mathrm{l}$ of the extraction was assessed using a highperformance lipid chromatography (HPLC) system, which comprised of the 2707 Autosampler (Waters Corporation, Milford, MA, USA), 600 Controller and Pump (Waters Corporation), reverse phase HPLC column $(4.6 \times 100 \mathrm{~mm}, 2 \mu \mathrm{m}$ macropores in diameter; 1.02129.0001; Merck KGaA) and 2489 UV/Visible Detector (Waters Corporation). HPLC was performed under a linear gradient of acetonitrile:water from 40:60 to $95: 5(\mathrm{v} / \mathrm{v})$ within $15 \mathrm{~min}$ at a flow rate of $0.8 \mathrm{ml} / \mathrm{min}$ at $20^{\circ} \mathrm{C}$. Signal was detected at a single wavelength of $227 \mathrm{~nm}$. The encapsulation efficiency (EE) was calculated as follows: $\mathrm{EE}(\%)=\mathrm{PTX}$ concentration in the filtered liposomes / PTX concentration in the unfiltered liposomes $\mathrm{x} 100 \%$. The phosphorus in the liposome suspension was determined by Bartlett assay (31). The lipo-PTX preparations were stored at $4^{\circ} \mathrm{C}$ and the stability during storage was determined by monitoring PTX content within the liposome suspension. Blank liposomes 
and Rh-DPPE-containing liposomes served as controls and were fabricated with SPC:cholesterol:mPEG-DSPE and SPC: cholesterol:mPEG-DSPE:Rh-DPPE at molar ratios of 97:2:1 and 97:2:0.8:0.2 to match lipo-PTX in each experiment.

For the preparation of non-extruded lissamine rhodamine $\mathrm{B}$ anchored liposomes containing OG488-PTX, Rh-DPPE and OG488-PTX were added at the same time as lipid materials. The dry lipid film was hydrated in PBS, without homogenization by freeze-and-thaw cycles and extrusion.

Characterization of liposomes. The mean size and zeta potential of lipo-PTX were detected by dynamic light scattering (Malvern Zetasizer Nano ZS; Malvern Instruments Ltd., Malvern, UK). The lipo-PTX samples were submitted to cryo-transmission election microscopy (Cryo-TEM) at the Core Facility, Department of Academic Affairs and Instrument Service at Academia Sinica (Taipei, Taiwan), to observe structural features (30).

In vitro drug release. The PTX release profiles from Taxol and lipo-PTX formulations were investigated according to a dialysis bag diffusion method. Briefly, $1 \mathrm{ml}$ Taxol or lipo-PTX $(1 \mathrm{mg} / \mathrm{ml})$ was placed in a dialysis tube (molecular weight cut-off 6-8 kDa; 71509-3; Merck KGaA) and immersed into $40 \mathrm{ml}$ release medium (PBS, $\mathrm{pH} 7.4$ or 5.0). The entire system was incubated at $37^{\circ} \mathrm{C}$ with orbital agitation at $150 \mathrm{rpm}$. The concentration of PTX remaining in the dialysis tube at predetermined time intervals $(0,0.5,1,2,4,6,8,24,48,72,96$ and $168 \mathrm{~h}$ ) was measured by HPLC.

Pharmacokinetic assay. NOD/SCID mice were intravenously injected with Taxol or lipo-PTX at $20 \mathrm{mg} / \mathrm{kg}$ (n=6/group). Blood samples were withdrawn into BD Microtainer ${ }^{\circledR}$ blood collection tubes with EDTA (365974; BD Biosciences, Franklin Lakes, NJ, USA) at various time points after injection $(0,0.25,0.5,1,2,4$, $6,8,24$ and $30 \mathrm{~h}$ ). Total blood samples were then centrifuged at $3,000 \mathrm{x}$ for $10 \mathrm{~min}$ at $4^{\circ} \mathrm{C}$ to obtain plasma samples. To remove the interfering substances, plasma protein was precipitated with 10X acetonitrile. The solution was agitated for $1 \mathrm{~h}$ to extract PTX from plasma completely. PTX dissolved in acetonitrile was quantified by HPLC (Waters Corporation).

Cell culture. Human breast cancer cell lines (MDA-MB-231, SKBR3 and MCF-7), human lung cancer cell lines (A549, H460 and CL1-5) and a human ovarian cancer cell line (SKOV3) were purchased from the American Type Culture Collection (ATCC; Manassas, VA, USA). Human liver cancer cell lines, Mahlavu and SK-HEP-1, were kindly provided by Dr M. Hsiao (Genomic Research Center, Academia Sinica). MDA-MB-231 cells were grown in DMEM/F12 medium supplemented with 10\% FBS. SKBR3, MCF-7, A549, H460 and CL1-5 cells were cultured in RPMI-1640 supplemented with $10 \%$ FBS. SKOV3, Mahlavu and SK-HEP-1 cells were maintained in DMEM supplemented with 10\% FBS. All cells were maintained in the appropriate medium containing $100 \mathrm{U} / \mathrm{ml} \mathrm{P} / \mathrm{S}$ and were incubated in a humidified atmosphere containing $5 \% \mathrm{CO}_{2}$ at $37^{\circ} \mathrm{C}$, according to the ATCC protocols.

Cellular uptake. The cellular internalization of liposomal contents was determined by quantifying the fluorescence intensity of lissamine rhodamine B within cells, which was conjugated to DPPE (Rh-DPPE) and inserted in the liposomes. MDA-MB-231 and SKOV3 cells were seeded onto 24-well plates at a density of $8 \times 10^{4}$ cells/well and were cultured for $24 \mathrm{~h}$ at $37^{\circ} \mathrm{C}$. The cells were then treated with Rh-DPPE-containing liposomes at a final Rh-DPPE concentration of 0, 2, 5, 10 and $20 \mu \mathrm{M}$ for $2 \mathrm{~h}$. After washing with cold PBS twice, cells were incubated with 0.1 M glycine ( $\mathrm{pH}$ 2.2) (50046; Sigma-Aldrich; Merck $\mathrm{KGaA}$ ) on ice for $5 \mathrm{~min}$ to remove residual liposomes from the cell surface. The cells were harvested by scraping with $1 \%$ Triton-X 100/PBS after washing with PBS twice. The fluorescence intensity in the cells was measured using a spectrofluorometer (SpectraMax M5, Molecular Devices) at excitation $520 \mathrm{~nm} / \mathrm{emission} 570 \mathrm{~nm}$.

In vitro cellular cytotoxicity. Cells were seeded onto a 96-well culture plate at $5 \times 10^{3}$ cells/well and were incubated with culture medium containing $5 \% \mathrm{FBS}$ overnight at $37^{\circ} \mathrm{C}$. Cells were then treated with two-fold serially diluted Taxol and lipo-PTX at doses ranging between 0.195 and $50 \mathrm{nM}$ for $48 \mathrm{~h}$. The equal amount of phosphorus in empty liposome suspension was used as a lipid toxicity control. After being washed with PBS twice, cells were incubated with serum-free medium containing $0.5 \mathrm{mg} / \mathrm{ml} \mathrm{MTT}$ for $4 \mathrm{~h}$ at $37^{\circ} \mathrm{C}$. Subsequently, the medium was removed, and purple crystals within the cells were dissolved with dimethyl sulfoxide. Cell viability was determined by measuring absorbance at $540 \mathrm{~nm}$.

In vivo antitumor efficacy. The flanks of NOD/SCID mice were subcutaneously (s.c.) injected with $2 \times 10^{6}$ cells (MDA-MB-231, SKOV3 or SK-HEP-1). The tumor volume was calculated as follows: Length $\mathrm{x}$ (width) ${ }^{2} \times 0.52$. When the average tumor volume reached $70-100 \mathrm{~mm}^{3}$, mice were randomized into different groups: Taxol, Abraxane, lipo-PTX and PBS ( $\mathrm{n}=5-11 /$ group). The drugs ( $15 \mathrm{or} 30 \mathrm{mg} / \mathrm{kg}$ PTX formulations) were intravenously administered every 4 days for a total of four injections. PBS was injected into the negative control group. The tumor inhibition rate was defined as follows: $100 \%$ - (tumor volume upon PTX injection/tumor volume of control group) x $100 \%$.

In vivo biodistribution of PTX. The flanks of NOD/SCID mice were injected (s.c.) with 107 MDA-MB-231 cells. Tumor volume was calculated as follows: Length $\mathrm{x}$ (width) ${ }^{2} \times 0.52$. When the average tumor volume reached $200-300 \mathrm{~mm}^{3}$, mice were randomized into four groups: PBS, Taxol, Abraxane and lipo-PTX (n=3/group). Mice were intravenously injected once with $15 \mathrm{mg} / \mathrm{kg}$ Taxol, Abraxane or lipo-PTX. Vital organs, including the brain, heart, liver, kidney and spleen, as well as tumors were harvested $2 \mathrm{~h}$ post-injection, and were homogenized in distilled water using a MagNA Lyser Instrument (Roche Diagnostics, Basel, Switzerland). PTX in the organs was extracted using $40 \%$ acetonitrile in distilled water and the samples were evaluated by HPLC (Waters Corporation).

In vivo toxicity assay. ICR mice were randomized into four groups ( $n=12 /$ group), and were intravenously injected with $15 \mathrm{mg} / \mathrm{kg}$ Taxol, Abraxane, lipo-PTX or PBS every 4 days for a total of four injections. A total of $1 \mathrm{~h}$ after the last injection, whole blood, which was collected in BD Microtainer ${ }^{\circledR}$ 
blood collection tubes (BD Biosciences) was supplied to Taiwan Mouse Clinic (Taipei, Taiwan) for blood cell count using the IDEXX ProCyte Dx Hematology Analyzer (IDEXX Laboratories, Inc., Westbrook, MA, USA).

For blood biochemistry examination, $24 \mathrm{~h}$ after the last injection, whole blood was collected in BD Microtainer ${ }^{\circledR}$ tubes (BD Biosciences) and was centrifuged at 3,000 x g for $10 \mathrm{~min}$ at $4^{\circ} \mathrm{C}$ to obtain plasma samples. Plasma samples were supplied to Taiwan Mouse Clinic to determine blood $\mathrm{Ca}^{2+}$ concentration, alanine aminotransferase (ALT), aspartate aminotransferase (AST), total bilirubin (TBIL), blood urea nitrogen (BUN) and uric acid (UA) using the Fuji Dri-Chem 4000i (Fujifilm, Tokyo, Japan).

For histological analysis, $24 \mathrm{~h}$ after the last intravenous treatment, major organs, including the brain, heart, liver, kidney and spleen were harvested, fixed in $10 \%$ neutrallybuffered formalin (3.7\% formaldehyde, $0.029 \mathrm{M} \mathrm{KH}_{2} \mathrm{PO} 4$, and $0.037 \mathrm{M} \mathrm{K}_{2} \mathrm{HPO}_{4}$ in distilled-deionized water) overnight at room temperature, and were then dehydrated in an ascending series of alcohol. The specimens were then immersed in xylene (Merck KGaA) and embedded in paraffin (39601006; Leica Biosystems Richmond, Inc., Richmond, IL, USA). Sections (size, $4 \mu \mathrm{m}$ ) were cut with a microtome (Olympus Cut 4055; Olympus Corporation, Tokyo, Japan) and mounted on slides. The slides then were subjected to hematoxylin and eosin staining. Briefly, the sections were deparaffinized in xylene and rehydrated in a descending series of alcohol. Subsequently, the sections were stained with Gill's hematoxylin (GHS132; Sigma-Aldrich; Merck KGaA) for $15 \mathrm{~min}$. After washing under running water for $15 \mathrm{~min}$, the sections were stained with $0.5 \%$ eosin Y (230251; Sigma-Aldrich; Merck KGaA) for $2 \mathrm{~min}$. Finally, the stained sections were dehydrated and mounted with Permount (Thermo Fisher Scientific, Inc.), and examined by light microscopy. All histopathological images were captured with an Olympus BX53 microscope (Olympus Corporation) and DP73-BSW image acquisition software (Olympus Corporation).

ECG recordings. The ECG recordings were performed noninvasively on anesthetized mice in lead II using PowerLab 8/30 and Animal Bio Amp (ADInstruments Pty Ltd., New South Wales, Australia) at the Taiwan Mouse Clinic Center (Taipei, Taiwan). The ECG was recorded continuously from $5 \mathrm{~min}$ before to 15 min after intravenous PTX administration in all individual ICR mice ( $\mathrm{n}=9$ /group). Heart rate, PR interval, RR interval, QRS interval and QT interval were analyzed using LabChart software version 8.1 (ADInstruments Pty Ltd.). The corrected QT (QTc) interval represented the QT interval normalized for heart rate using Bazett's formula: $\mathrm{QTc}=$ measured $\mathrm{QT}$ interval $/ \sqrt{ } \mathrm{RR}$ interval.

Statistical analysis. Data are presented as the means \pm standard deviation or standard error of the mean of at least three independent experiments. GraphPad Prism software version 5 (GraphPad Software, Inc., La Jolla, CA, USA) was used for statistical analysis. Two-tailed unpaired Student's t-test was used for comparisons between two groups. One-way analysis of variance (ANOVA) followed by Student-Newman-Keuls test and two-way ANOVA followed by Bonferroni post hoc test were used for comparisons among three or more groups.
Table I. Characteristics of liposomal formulation.

\begin{tabular}{lrr}
\hline Variable & Liposome & \multicolumn{1}{c}{ lipo-PTX } \\
\hline Average particle size (nm) & $127.98 \pm 3.35$ & $123.12 \pm 4.18$ \\
Polydispersity index & $0.12 \pm 0.02$ & $0.12 \pm 0.01$ \\
Zeta potential (mV) & $-17.52 \pm 0.64$ & $-15.42 \pm 1.40$ \\
PTX encapsulation & - & $94.16 \pm 1.85$ \\
efficiency (\%) & & \\
No. of PTX molecules in & & $2.30 \times 10^{4}$ \\
a liposome & & \\
\end{tabular}

Data are presented as the means \pm standard deviation. PTX, paclitaxel; lipo-PTX, novel liposomal PTX.

$\mathrm{P}<0.05$ was considered to indicate a statistically significant difference.

\section{Results}

Development of a homogenous and stable lipo-PTX formulation. Physical characteristics of formulations, including particle size and surface charge, have a marked impact on the bioavailability and solubility of therapeutics. The mean particle size of liposomes and lipo-PTX used in the present study was $\sim 125 \mathrm{~nm}$ and the polydispersity index was 0.12 . No substantial differences in size distribution or mean size were detected between the control liposomes and lipo-PTX. The nanoparticles were mildly negatively charged (surface potential: $-15.42 \pm 1.40 \mathrm{mV}$, Table I and Fig. 1A). These measurements indicated that this liposomal preparation was homogeneous and suitable for penetration through leaky tumor vasculature, while evading clearance by phagocytic systems. With regards to therapeutic payload, EE was $94 \%$, and $2 \times 10^{4}$ molecules of PTX were estimated to be loaded per vesicle (Table I).

Cryo-TEM imaging demonstrated that the nanoparticles were spherical liposomes, composed of lipid bilayers (Fig. 1B). Co-localization of OG488-PTX and Rh-DPPE on non-extruded liposomes was confirmed by confocal microscopy. Furthermore, the PTX payload was harbored within the lipid membrane and not the aqueous core of liposomes (Fig. 1C). Subsequently, the stability of lipo-PTX was evaluated over the course of 300 days. The PTX concentration in the liposome suspension was not significantly altered within 250 days at $4^{\circ} \mathrm{C}$ (Fig. 1D). These results indicated that the liposomes were stable and possessed a reasonable shelf life for future clinical application.

Lipo-PTX is bioequivalent to Taxol in vivo. Premature drug release in serum and tissue fluid may hamper the therapeutic effects of a drug and cause undesirable toxicity to normal tissues. The present study monitored the release profile of lipo-PTX and Taxol at physiological pH (Fig. 2A). At pH 7.4, only $16 \%$ of PTX was released from lipo-PTX within $24 \mathrm{~h}$, whereas $45 \%$ of PTX was released from Taxol in the same time period. Taxol achieved 50\% PTX release at $34 \mathrm{~h}$; conversely, the amount of PTX released from lipo-PTX was only $47 \%$ after $168 \mathrm{~h}$ incubation in PBS at $\mathrm{pH}$ 7.4. These results 

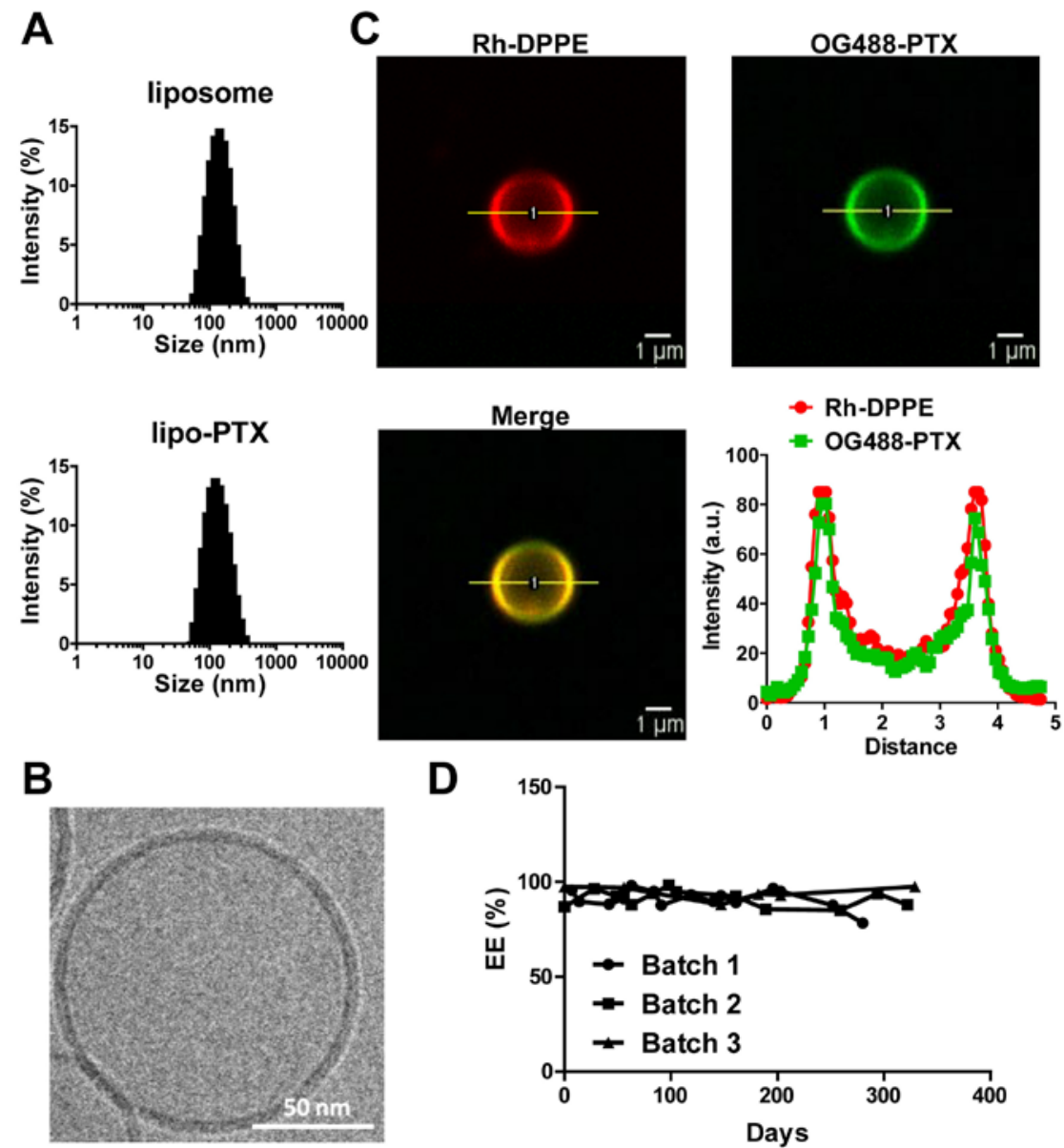

Figure 1. Physicochemical properties of liposomal formulations. (A) Particle size distribution of liposomes and lipo-PTX. (B) Cryo-transmission electron microscopy images of lipo-PTX. (C) Confocal fluorescence image of a single liposome containing both Rh-DPPE (red) and OG488-PTX (green), and their corresponding fluorescence intensity profiles. (D) Concentration profiles of lipo-PTX at various time-points. EE, encapsulation efficiency; lipo-PTX, novel liposomal PTX; OG488, Oregon Green ${ }^{\circledR}$ 488; PTX, paclitaxel; Rh-DPPE, lissamine rhodamine B-conjugated 1,2-dipalmitoyl-sn-glycero-3-phosphoethanolamine- $\mathrm{N}$-(lissamine rhodamine B sulfonyl) (ammonium salt).

A

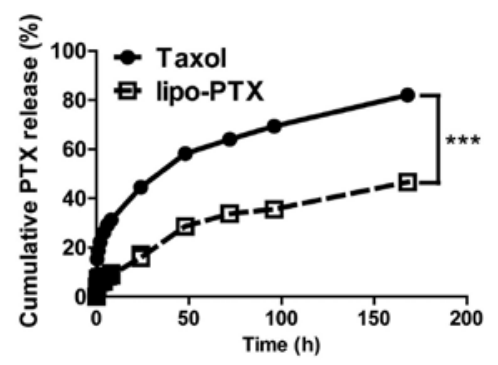

B

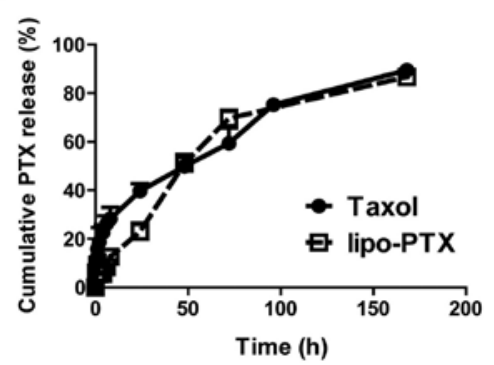

Figure 2. In vitro PTX release profiles. Drug release profiles of PTX from Taxol and lipo-PTX in (A) pH 7.4 or (B) pH 5.0 PBS at $37^{\circ} \mathrm{C}$. lipo-PTX, novel liposomal PTX; PTX, paclitaxel. Data are presented as the means \pm standard deviation. ${ }^{* * *} \mathrm{P}<0.001$, two-way analysis of variance followed by Bonferroni post hoc test.

suggested that lipo-PTX is significantly more stable than the Taxol formulation and that collateral toxicity to normal tissue might be alleviated due to reduced premature release of PTX.

Nanoparticles taken up by cells are expected to fuse with endosomes and lysosomes, where the contents will be degraded in an acidic microenvironment with a $\mathrm{pH}$ value near $5.0(32,33)$. The profile of drug release from Taxol at $\mathrm{pH} 5.0$ was comparable to that at $\mathrm{pH}$ 7.4. However, PTX release from lipo-PTX exhibited a 1.5- to 2-fold increase following $48 \mathrm{~h}$ in an acidic environment
(pH 5.0) when compared with at pH 7.4 (48 h, 51 vs. 29\%; 72 h, 70 vs. $34 \%$; 168 h, 87 vs. $47 \%$ ) (Fig. 2B). These results indicated that lipo-PTX is a $\mathrm{pH}$-sensitive drug delivery platform, which is stable at physiological $\mathrm{pH}$. However, the efficiency of drug release is enhanced in acidic environments, such as those that exist in intracellular lysosomes and intratumoral stroma.

The present study also compared the pharmacokinetics of Taxol and lipo-PTX. Both PTX formulations were administered intravenously to NOD/SCID mice, and the 


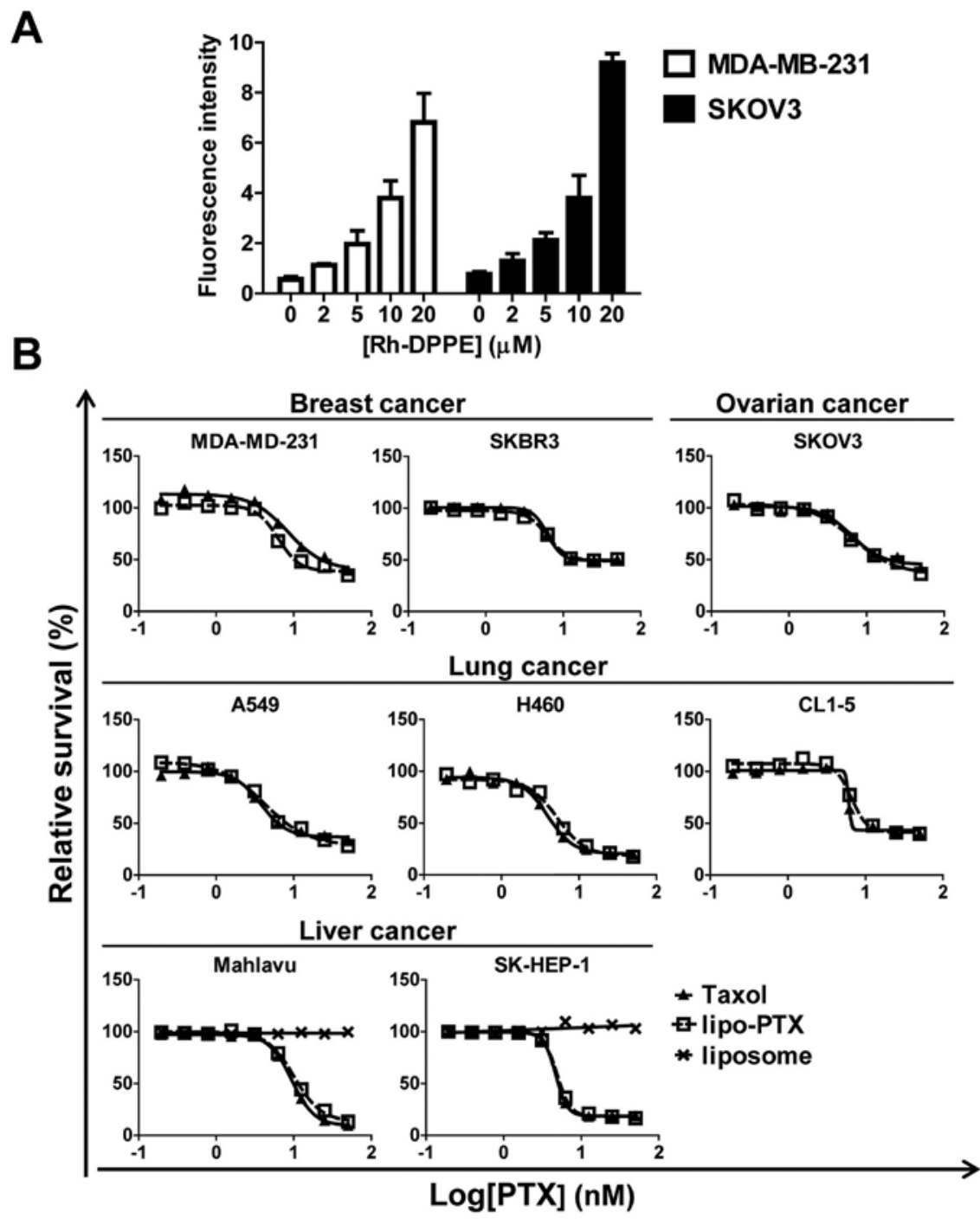

Figure 3. In vitro cellular cytotoxicity. (A) Cellular internalization was measured by fluorescence intensity of Rh-DPPE-liposomes. (B) In vitro cytotoxicity profiles of Taxol and lipo-PTX, as determined by the MTT assay. Data are presented as the percentage of viable cells compared with the untreated group. Data are expressed as the means \pm standard deviation. lipo-PTX, novel liposomal PTX; PTX, paclitaxel; Rh-DPPE, lissamine rhodamine B-conjugated 1,2-dipalmitoyl-sn-glycero-3-phosphoethanolamine- $\mathrm{N}$-(lissamine rhodamine B sulfonyl) (ammonium salt).

time-dependent plasma PTX concentration profiles were determined by HPLC. As shown in Table II, PTX concentration in the plasma was continually and quickly diminished following Taxol administration, and dropped to the basal level after $10 \mathrm{~h}$. The area under the curve (AUC) $)_{0 \rightarrow 24}$ for Taxol injection was $53.24 \mathrm{mg} \cdot \mathrm{h} / \mathrm{l}$, and the half-life $\left(\mathrm{t}_{1 / 2}\right)$ was $1.10 \mathrm{~h}$. Total clearance was $0.38 \mathrm{l} / \mathrm{h} / \mathrm{kg}$, and the elimination constant for Taxol was $0.63 \mathrm{~h}-1$ (Table II). All of the pharmacokinetic parameters were consistent with the results from a previous study in mice (34). Although the blood PTX concentration and $\mathrm{AUC}_{0 \rightarrow 24}(89.12 \mathrm{mg} \cdot \mathrm{h} / \mathrm{l})$ in the lipo-PTX-administered group were slightly higher than those in the Taxol group, lipo-PTX only slightly extended the circulation time for PTX in the blood $\left(\mathrm{t}_{1 / 2}=1.73 \mathrm{~h}\right)$ compared with Taxol $\left(\mathrm{t}_{1 / 2}=1.10 \mathrm{~h}\right)$. Consequently, there were no significant differences in pharmacokinetic parameters between lipo-PTX and Taxol (Table II), thus indicating that lipo-PTX was bioequivalent to Taxol in vivo.

Antineoplastic efficacy of lipo-PTX is comparable to that of commercial PTX formulations in vitro and in vivo. To confirm
Table II. Pharmacokinetic parameters of PTX observed in NOD/SCID mice after intravenous injection administration of $20 \mathrm{mg} / \mathrm{kg}$ of Taxol and lipo-PTX.

\begin{tabular}{lccccc}
\hline Therapy & $\begin{array}{c}\text { Dose } \\
(\mathrm{mg} / \mathrm{kg})\end{array}$ & $\begin{array}{c}\mathrm{AUC}_{0 \rightarrow 24} \\
(\mathrm{mg} \cdot \mathrm{h} / \mathrm{l})\end{array}$ & $\begin{array}{c}\mathrm{K} \\
\left(\mathrm{h}^{-1}\right)\end{array}$ & $\begin{array}{c}\mathrm{t}_{1 / 2} \\
(\mathrm{~h})\end{array}$ & $\begin{array}{c}\mathrm{CL} \\
(\mathrm{l} / \mathrm{h} / \mathrm{kg})\end{array}$ \\
\hline Taxol & 20 & 53.17 & 0.63 & 1.10 & 0.38 \\
lipo-PTX & 20 & 89.12 & 0.40 & 1.73 & 0.22 \\
\hline
\end{tabular}

Data were presented as the mean from six independent mice in each group. $\mathrm{AUC}_{0 \rightarrow 24}$, area under curve from 0 to $24 \mathrm{~h}$; CL, total clearance rate; $\mathrm{K}$, elimination rate constant; lipo-PTX, novel liposomal PTX; PTX, paclitaxel; $\mathrm{t}_{1 / 2}$, elimination half-life.

that lipo-PTX would be taken up by cancer cells, Rh-DPPE was used to quantify the level of cellular internalization. The intracellular fluorescence in MDA-MB-231 and SKOV3 cells was dose-dependently increased following incubation with 

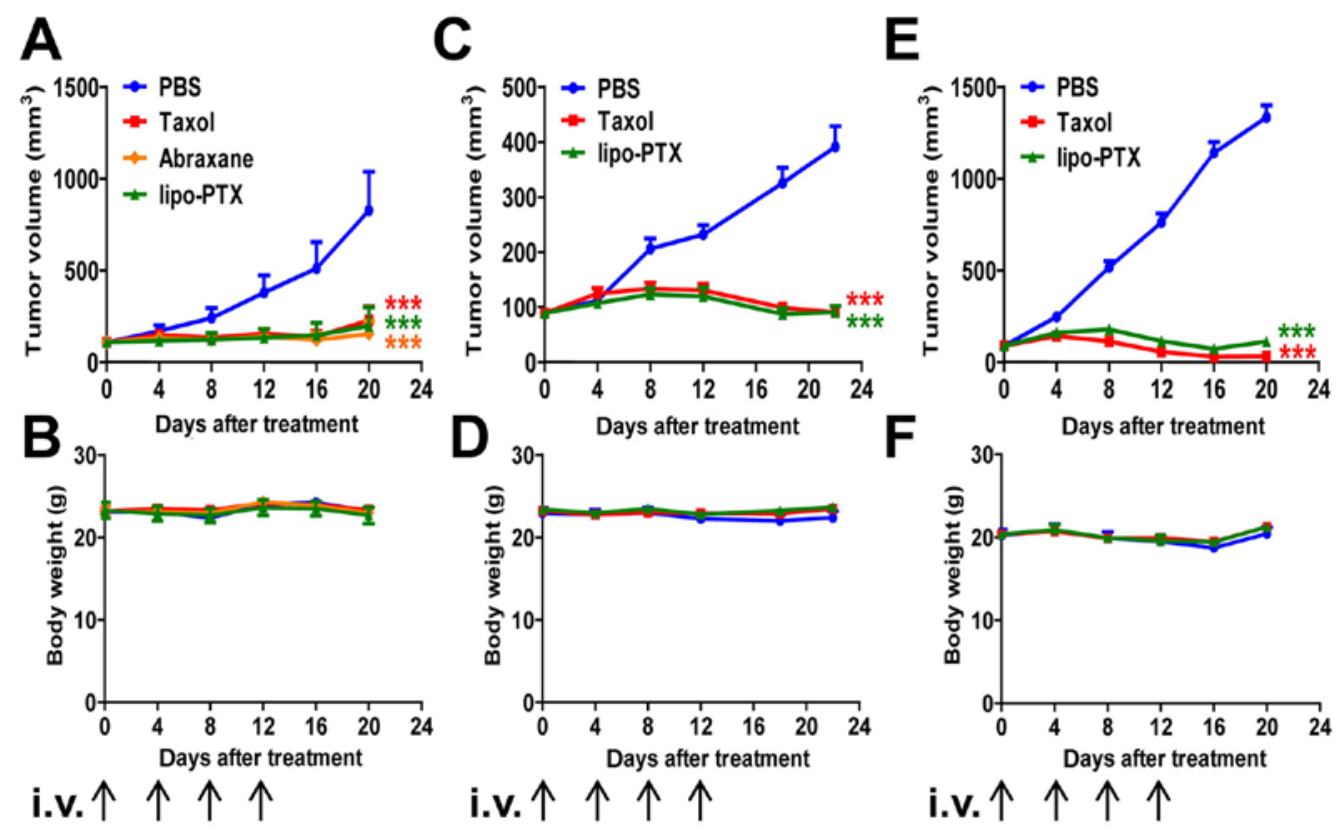

i.v. $\uparrow \uparrow \uparrow \uparrow$
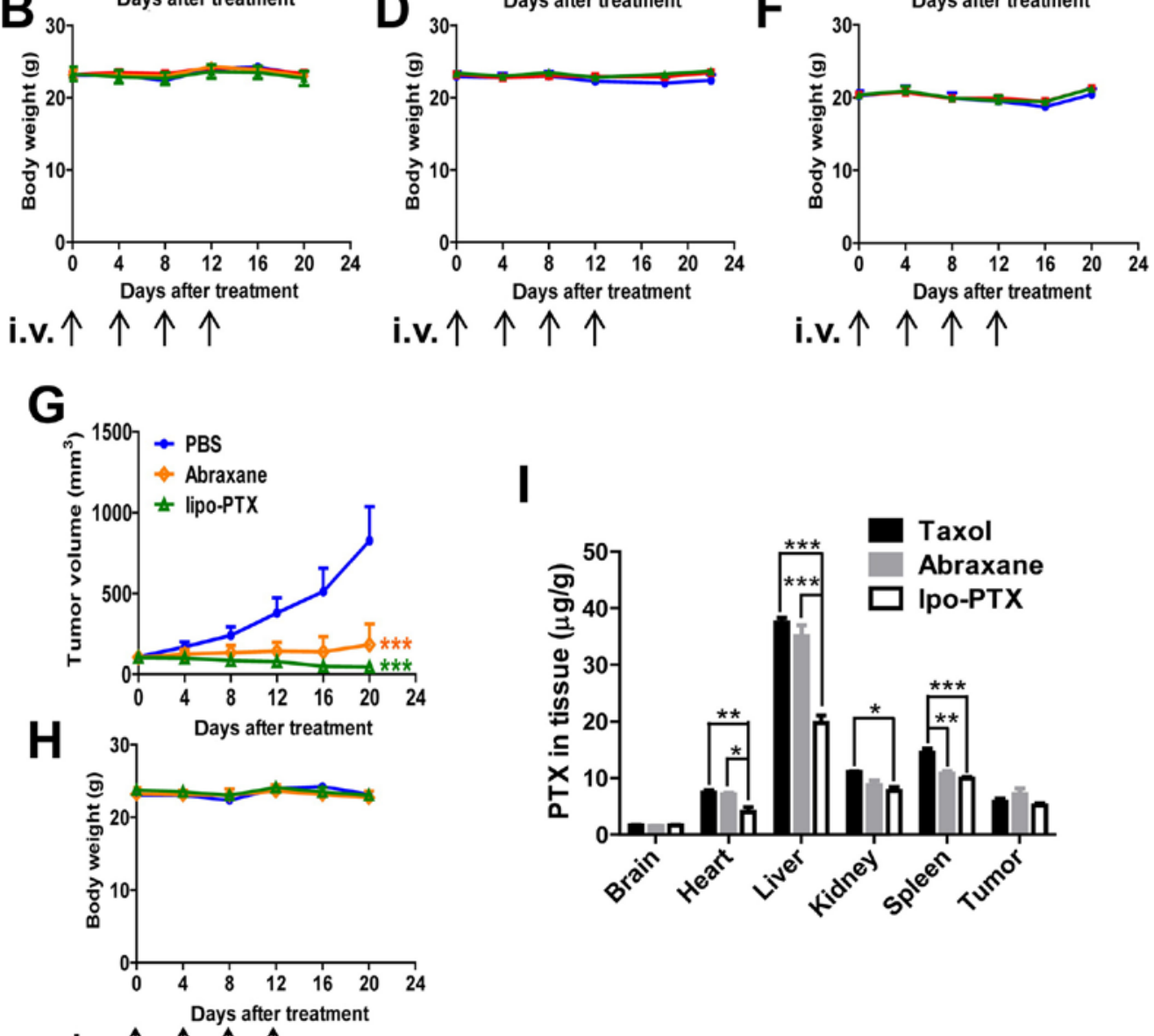

i.v. $\uparrow \uparrow \uparrow \uparrow$

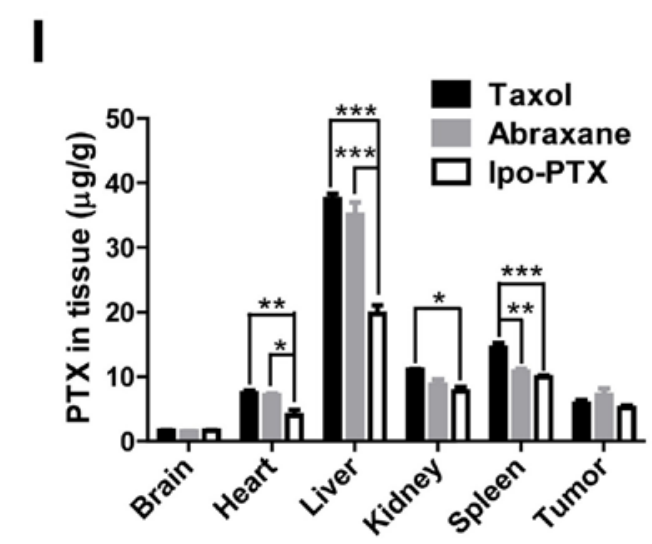

Figure 4. Therapeutic efficacy of liposomal PTX in xenograft models. (A-H) Mouse xenograft models of (A, B, G and H) MDA-MB-231 human breast cancer cells, (C and D) SKOV3 human ovarian cancer cells or (E and F) SK-HEP-1 human liver cancer cells; mice were intravenously injected four times with Taxol, Abraxane or lipo-PTX at 15 or $30 \mathrm{mg} / \mathrm{kg}$ every 4 days. PBS was used as a negative control. (A, C, E and G) Tumor volume and (B, D, F and H) body weight within each group were determined. (A and B, $15 \mathrm{mg} / \mathrm{kg}, \mathrm{n}=6 ; \mathrm{C}$ and D, $15 \mathrm{mg} / \mathrm{kg}, \mathrm{n}=11 ; \mathrm{E}$ and F, $15 \mathrm{mg} / \mathrm{kg}, \mathrm{n}=8 ; \mathrm{G}$ and H, $30 \mathrm{mg} / \mathrm{kg}$, $\mathrm{n}=5$ ). Data are presented as the means \pm standard error of the mean. ${ }^{* * *} \mathrm{P}<0.001$ compared with the PBS group, two-way analysis of variance followed by Bonferroni post hoc test. (I) PTX distribution in MDA-MB-231-bearing mice $2 \mathrm{~h}$ after treatment with $15 \mathrm{mg} / \mathrm{kg}$ Taxol, Abraxane or lipo-PTX. Data are presented as the means \pm standard error of the mean from three independent mice in each group. ${ }^{*} \mathrm{P}<0.05,{ }^{* *} \mathrm{P}<0.01,{ }^{* * * *} \mathrm{P}<0.001$, two-way analysis of variance followed by Bonferroni post hoc test. lipo-PTX, novel liposomal PTX; PTX, paclitaxel.

liposomes (Fig. 3A), thus indicating that the cells took up the liposomal nanoparticles. In addition, the antineoplastic action of lipo-PTX was detected in cell cultures. The results indicated that lipo-PTX markedly suppressed cell proliferation in various cancer cell lines. The half maximal inhibitory concentration $\left(\mathrm{IC}_{50}\right)$ values of lipo-PTX ranged between 4 . and $13.2 \mathrm{nM}$, and were similar to those of Taxol in each corresponding cell line (Fig. 3B). No cytotoxicity was discernible when liver cancer cells were treated with empty liposomes as a negative control. Taken together, these data suggested that the therapeutic effects of lipo-PTX resulted from the PTX payload, instead of the liposomal vehicle, and that its antineoplastic action was comparable to that of CrEL-containing Taxol.

The present study further compared the therapeutic efficacy of lipo-PTX to Taxol in various subcutaneous xenograft murine models (Fig. 4A-H). Body weight was recorded and served as a gross indicator of systemic toxicity. As shown in Fig. 4A, MDA-MB-231 xenografts were sensitive to treatment with both Taxol and lipo-PTX, and tumor size was markedly reduced following PTX infusion. Lipo-PTX administration suppressed the tumor burden of MDA-MB-231 xenograft 

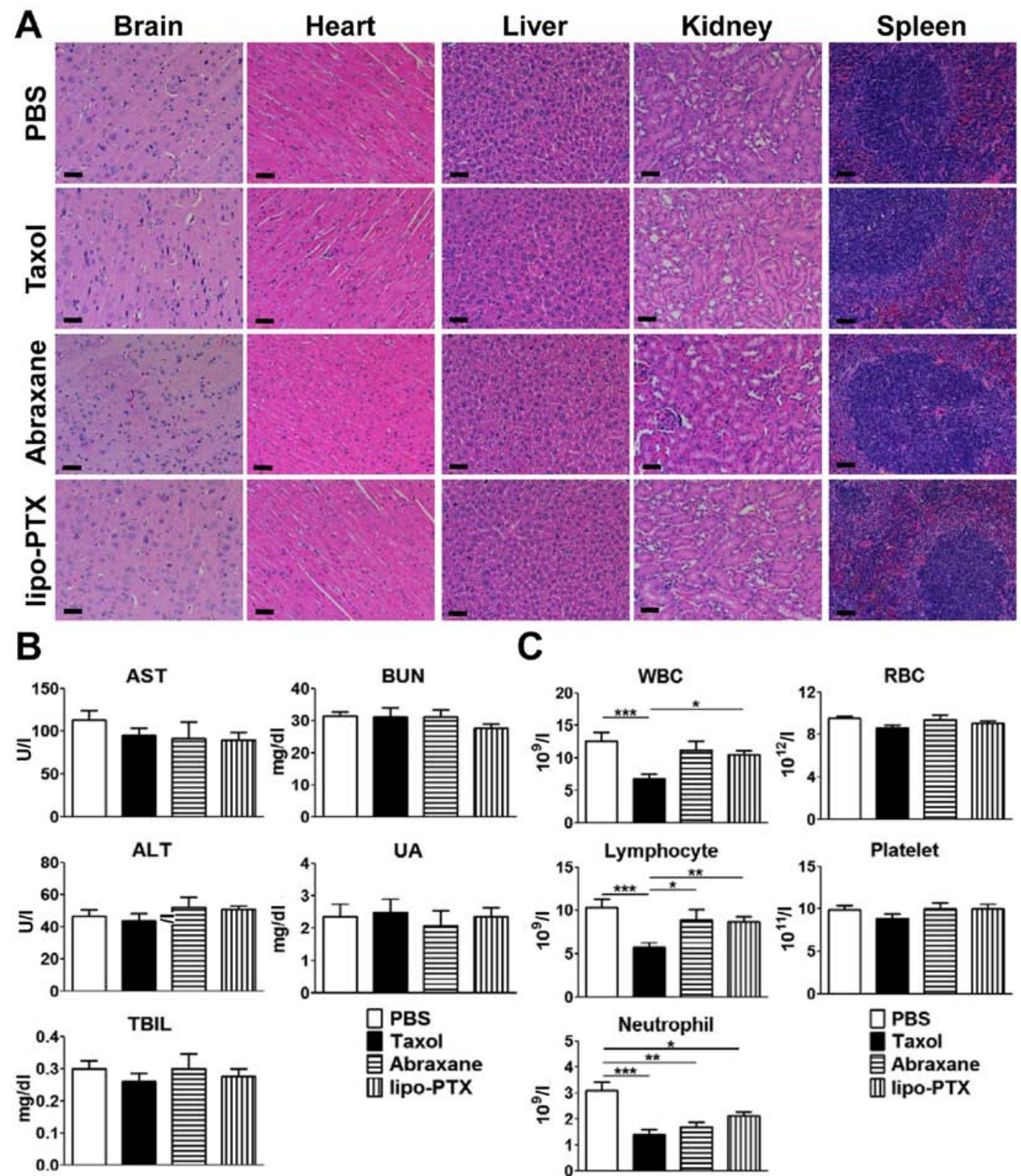

Figure 5. Evaluation of potential toxicity of lipo-PTX. Healthy tumor-free ICR mice were intravenously injected four times with Taxol or lipo-PTX (15 mg/kg) every 4 days. PBS was used as a negative control. Blood and organs were harvested $1 \mathrm{~h}$ after the last treatment. (A) Histopathology of vital organs, (B) serum chemistry levels and $(\mathrm{C})$ blood counts are shown. Scale bars, $50 \mathrm{~nm}$. Data are presented as the means \pm standard deviation $(\mathrm{n}=12) .{ }^{*} \mathrm{P}<0.05,{ }^{* *} \mathrm{P}<0.01,{ }^{* * * *} \mathrm{P}<0.001$, one-way analysis of variance followed by Student-Newman-Keuls test. ALT, alanine aminotransferase; AST, aspartate aminotransferase; BUN, blood urea nitrogen; lipo-PTX, novel liposomal PTX; PTX, paclitaxel; RBC, red blood cells; TBIL, total bilirubin; UA, uric acid; WBC, white blood cells.

mice slightly more efficiently than Taxol did (inhibition rate on day 20, 76 vs. $72 \%$ ). In SKOV3 xenograft mice, lipo-PTX exhibited a comparable therapeutic effect to Taxol (inhibition rate on day 26, 82 vs. 84\%) (Fig. 4C). However, lipo-PTX exhibited a slightly inferior antineoplastic effect compared with Taxol on SK-HEP-1 xenografts (inhibition rate on day 20, 92 vs. 98\%) (Fig. 4E). In addition, the therapeutic effects of lipo-PTX were compared with those of Abraxane in MDA-MB-231 xenografts. The inhibitory rate of lipo-PTX $(15 \mathrm{mg} / \mathrm{kg}$ ) was slightly lower than that of Abraxane (inhibition rate on day 20,76 vs. $81 \%$, Fig. $4 \mathrm{~A}$ ); however, administration with $30 \mathrm{mg} / \mathrm{kg}$ lipo-PTX suppressed tumor growth more efficiently than Abraxane (inhibitory rate, 94 vs. 77\%, Fig. 4G). The effects of empty liposomes were not detected in vivo, because the intention was to compare the novel liposome formulation with existing formulations. Overall, lipo-PTX exhibited a therapeutic effect that was superior or equivalent to that of Taxol and Abraxane in numerous murine subcutaneous xenograft models. No significant systemic toxicity was observed in any group, as the body weight of the mice was stable (Fig. 4B, D, F and H).

Lipo-PTX has reduced side effects compared with Taxol or Abraxane. Although no significant alterations in body weight were detected in mice treated with Taxol, Abraxane or lipo-PTX (Fig. 4B, D, F and H), HSRs were observed upon administration of Taxol, but not lipo-PTX (data not shown). The reactions were characterized by loss of motility, angioedema, dyspnea, syncope and even mortality. To further investigate the systemic toxicity of lipo-PTX, PTX 

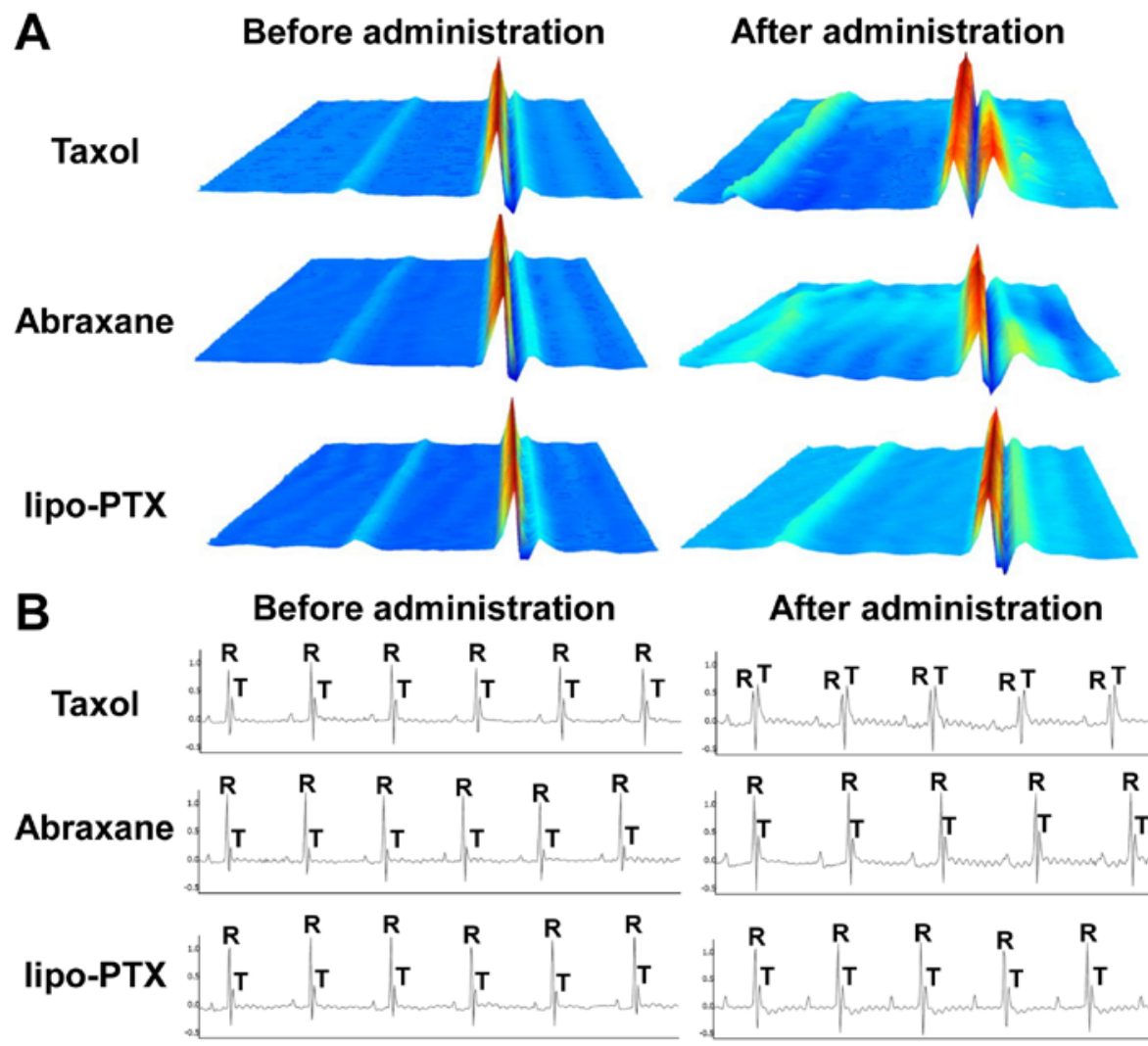

After administration
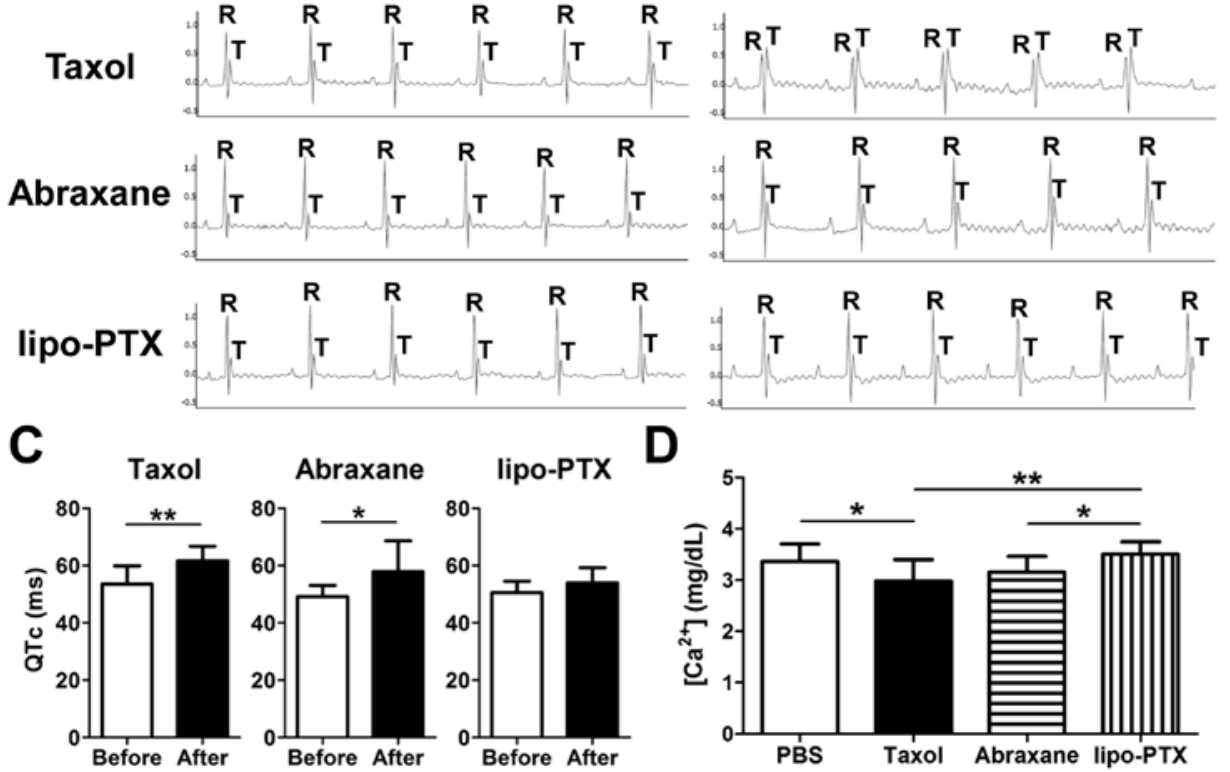

D

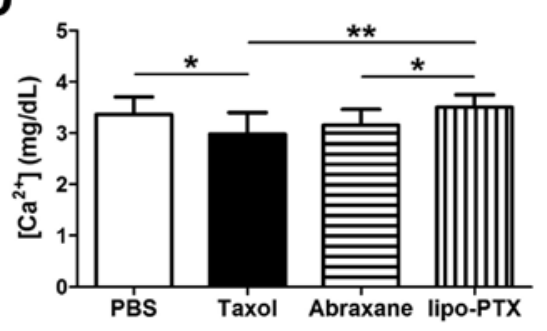

Figure 6. ECGs of ICR mice before and after PTX administration. (A) ECGs were recorded from anesthetized mice before and after injection of PTX solution $(15 \mathrm{mg} / \mathrm{kg})$. (B) ECG traces were recorded in 2-sec excerpts from anesthetized mice before and after injection of PTX solution (15 mg/kg). (C) QTc values before and after PTX administration. ${ }^{*} \mathrm{P}<0.05,{ }^{* *} \mathrm{P}<0.01$, two tail unpaired Student's t-test. (D) Calcium concentration in the blood following PTX administration. Data are presented as the means \pm standard deviation $(n=9) .{ }^{*} \mathrm{P}<0.05,{ }^{* *} \mathrm{P}<0.01$, one-way analysis of variance followed by Student-Newman-Keuls test. ECG, electrocardiogram; lipo-PTX, novel liposomal PTX; PTX, paclitaxel.

accumulation in major organs was examined $2 \mathrm{~h}$ after the injection of PTX formulations. If the tumor is too small, the blood supply to the tumor will be limited, making it difficult to measure the precise dosage and time for drug accumulation. When the tumor is too large, the center of the tumor will undergo necrosis, which will also impact the drug/tissue ratio (w/w) (30). Therefore, tumor volume at 200-300 $\mathrm{mm}^{3}$ was used to avoid problems associated with limited blood supply or necrosis. As shown in Fig. 4I, Taxol, Abraxane and lipo-PTX mainly accumulated in the liver, spleen and kidney. No evident histological abnormalities were noted in the vital organs, including the brain, heart, liver, kidney and spleen of any of the four groups (Fig. 5A). In addition, no significant elevation of liver damage indicators (serum ALT, AST, and TBIL) and kidney function markers (BUN and UA) was detected in any of the treatment groups (Fig. 5B). Taken together, these data indicated that no evident hepatic damage or renal impairment was present in any treatment group.
With regards to the suppression of hematopoiesis, Taxol administration markedly decreased the number of white blood cells, lymphocytes and neutrophils compared with in the PBS-treated group (Fig. 5C). Conversely, no significant myelosuppression was noted in groups treated with lipo-PTX or Abraxane, except for a consistent decrease in neutrophils in all three treatment groups. Nevertheless, the reduction in neutrophils was not as apparent in the lipo-PTX group as in the Taxol group. Furthermore, the lymphocyte and white blood cell populations of the lipo-PTX group were significantly higher compared with in the Taxol group, whereas the differences between lipo-PTX and Abraxane were not significant. Therefore, it may be concluded that lipo-PTX has lower hematopoietic toxicity than Taxol.

Taxol, Abraxane and lipo-PTX deposited similar amounts of PTX in the tumor tissues following treatment (Fig. 4I). Notably, there was more Taxol and Abraxane accumulation in heart than that of lipo-PTX. In a previous study, Taxol infusion was revealed to induce sinus bradycardia and other myocardial 
Table III. Comparative effectiveness of lipo-PTX.

\begin{tabular}{lccc}
\hline Variable & Taxol & Abraxane & lipo-PTX \\
\hline Therapeutic efficacy $^{\mathrm{a}}$ & +++ & +++ & +++ \\
Body weight change $^{\mathrm{a}}$ & - & - & - \\
Histological damage $^{\mathrm{a}}$ & - & - & - \\
Liver and kidney function loss $^{\mathrm{a}}$ & - & - & - \\
Lymphocytopenia $^{\mathrm{a}}$ & +++ & - & - \\
Neutropenia $^{\mathrm{a}}$ & ++ & + & - \\
Anemia $^{\mathrm{a}}$ & ++ & - & - \\
Thrombocytopenia $^{\mathrm{a}}$ & - & - & - \\
ECG abnormalities $^{\text {QTc, prolonged }}$ & +++ & ++ & + \\
Decreased blood Ca $^{\mathrm{b}}$ & ++ & + & - \\
\end{tabular}

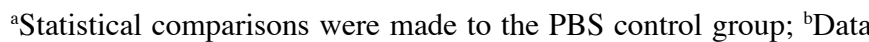
from before and after PTX treatment were statistically compared; 'Statistical comparisons were made to the lipo-PTX group. +++, significant difference $(\mathrm{P}<0.001) ;++$ and \#\#, moderately significant difference $(\mathrm{P}<0.01) ;+$ and $\#$, slight difference $(\mathrm{P}<0.05)$; -, no difference. ECG, electrocardiogram; lipo-PTX, novel liposomal PTX; PTX, paclitaxel.

disturbances (10). To further elucidate the cardiovascular impacts of drug administration, ECG traces were recorded and analyzed (Fig. 6A-C). Overall, ECG abnormalities were present in $100 \%$ (9/9) of mice injected with Taxol, whereas this phenomenon was only seen in $66 \%(6 / 9)$ of mice treated with Abraxane and 22\% (2/9) of mice treated with lipo-PTX. Notably, the irregularities in lipo-PTX-treated mice were transient and sustained for less than $3 \mathrm{~min}$, which was approximately one-fourth the duration observed in the Taxol group during the first 15 min after administration. Mice treated with Taxol and Abraxane exhibited abnormal ECG recordings, which included an irregular heartbeat and apparent QTc prolongation (Fig. 6A and C), indicating cardiac arrhythmia. Furthermore, a reduction in R-wave amplitude and an increase in T-wave amplitude were detected in the Taxol-treated mice (Fig. 6B). These irregularities indicated that Taxol may impair the strength of contractions, as previously reported (35). Although no apparent alterations were observed in the R- and T-wave amplitude following Abraxane infusion, an irregular RR interval and an increase in QTc, which suggested long QT syndrome, were detected (Fig. 6A and C). Conversely, there were no obvious aberrations in ECG recordings following lipo-PTX treatment.

Previous studies have demonstrated that Taxol impairs cardiac contraction via the acceleration of L-type calcium incurrent and a reduction in calcium signaling through the phosphoinositide signaling pathway (36-38). Therefore, calcium concentration in the serum was further measured. As shown in Fig. 6D, calcium concentration was significantly reduced in Taxol-treated mice. Lipo-PTX-treated mice, but not Abraxane-treated mice, exhibited a significantly increased calcium concentration compared with in the Taxol-treated group. These results suggested that lipo-PTX inflicts less collateral damage to normal tissue compared with CrEL-containing
Taxol or albumin-associated Abraxane; therefore, lipo-PTX may be more suitable for clinical use. The potential benefits of lipo-PTX compared with Taxol and Abraxane are summarized in Table III.

\section{Discussion}

One primary goal of successful cancer treatment regimens is to deliver sufficient amounts of drug to tumors while minimizing damage to normal tissues. Therefore, novel drug delivery systems are often generated from ongoing efforts to improve the selectivity and efficacy of antineoplastic drugs. Recently, many of these novel drug delivery systems comprise nanoparticles $(14,15)$, including lipid-based carriers, such as liposomes. Compared with conventional administration methods for chemotherapeutic agents, lipid- or polymer-based nanomedicines have the advantage of improving the pharmacological and therapeutic properties of cytotoxic drugs (39). The present study developed and optimized a novel liposome formulation of PTX, which exhibited high drug EE and liposome stability, and resulted in fewer side effects compared with commercial PTX formulations.

Numerous factors in the manufacturing of liposomes may affect the stability of the end product. These include the type of constitutive lipid, the presence of cholesterol and/or polyethylene glycol (PEG), and the ratio of lipid to therapeutic payload. PTX, which is hydrophobic, is not water-soluble and precipitates in aqueous solution. The present study used SPC, cholesterol and mPEG-DSPE in the liposome formulation. These materials are all amphipathic fatty acids, which were used due to their preferable lipophilic characteristics and ability to encapsulate PTX in the lipid bilayer. The outer layer of the liposome is hydrophilic, allowing suspension in aqueous solution. The bulky PEG head with highly hydrated groups on the liposome surface serves to sterically inhibit hydrophobic and electrostatic interactions with plasma proteins, decreasing recognition by the mononuclear phagocyte system (MPS). PTX, which is encapsulated in the lipid bilayer of the liposome, does not come into contact with the aqueous solution.

To determine the optimal formulation for desirable liposome properties, liposomes derived from saturated lipid acid (DSPC), monounsaturated lipid acid (egg PC) and polyunsaturated lipid acid (SPC) were analyzed. It was demonstrated that the loading efficiency of liposomes composed of DSPC was only 50\% that of SPC liposomes, and the vesicles broke up within 7 days. The stability of liposomes derived from monounsaturated lipid acid was slightly better, since nanoparticle rupture occurred on day 14 (data not shown). Conversely, the liposomes synthesized from SPC were stable for 328 days when stored at $4^{\circ} \mathrm{C}$, and they also possessed better loading efficiency for PTX. Sufficient space in the hydrophobic region of the lipid bilayers is necessary for high loading capacity of PTX in liposomal platforms (25). Addition of cholesterol can expand the inter-membrane space within the liposome; however, this supplement also increases the stiffness of the liposomes and diminishes their stability $(25,40,41)$. The present study revealed that the integrity of liposomes was sustained for only 90 days when the molar ratio of cholesterol was elevated from to 4 to $6 \%$, whereas the increase in loading efficiency was minimal (84 
and $85 \%$, respectively) (data not shown). Such a short duration of stability is unsuitable for clinical application. Surface PEGylation of liposomes is known to help particles evade premature clearance by the MPS, in order to prolong half-life in serum. However, these functions are gained at the expense of decreasing loading efficiency and stability of hydrophobic drugs $(24,25,42)$. In the present study, the PEG-containing (1\% molar ratio) formulation exhibited a shelf life of 328 days, whereas its EE of PTX was 94\%, which was far superior to previous PEGylated liposome formulations $(22,24)$. Although it is tempting to load the delivery system with as much drug as possible, the present study observed that the stability of the liposome was compromised if PTX was loaded at a molar ratio more than $2 \%$. The liposomes ruptured within 60 days when loaded at $3 \%$ and 1 day after encapsulation of $4 \%$ PTX (data not shown). Compared with previously published liposomal PTX formulations $(43,44)$, the newly developed lipo-PTX formulation exhibited much higher PTX loading efficiency. Notably, to the best of our knowledge, no publication has reported a liposomal formulation of PTX with such a long shelf life $(23,45-47)$.

The potential benefits of the lipo-PTX formulation compared with two commercial PTX formulations, Taxol and Abraxane. At present, there are two liposomal PTX formulations (EndoTAG-1 and LEP-ETU) in clinical use. EndoTAG-1, which targets the tumor vasculature instead of tumor cells $(48,49)$, is currently in phase III clinical trials. LEP-ETU was approved by the FDA in 2015 as an orphan drug for use in ovarian cancer treatment. However, its pharmacokinetic profile and therapeutic efficacy exhibits no significant benefits over Taxol $(50,51)$.

PTX administration has been associated with myocardial ischemia, infarction, arrhythmias and ECG abnormalities in patients; however, the mechanisms underlying cardiotoxicity are not well defined. It has previously been suggested that Taxol-induced arrhythmia is caused either directly by the effects of PTX on the Purkinje system or indirectly via CrEL-mediated histamine release (52). However, since ECG abnormalities are also common in patients treated with CrEL-free Abraxane infusion, it is most likely that the direct effects of PTX on cardiomyocytes are clinically important. In the case of Abraxane, the instability of the formulation in circulation may be considered a major risk for inducing ECG abnormalities. In a previous study, the albumin-PTX complex and unbound PTX were monitored in plasma following Abraxane injection. The unbound form comprised $6.4 \%$ of total drug in human plasma and this percentage did not vary with time (53). Notably, it has also been shown that Abraxane injection leads to greater PTX accumulation in the heart than Taxol, both 1 and 5 days after dosing (54). The present results regarding drug distribution indicated that there were no significant differences in PTX accumulation between Taxoland Abraxane-treated groups; however, lipo-PTX treatment reduced PTX accumulation in the heart compared with either Taxol or Abraxane administration. These results suggested that, compared with both Taxol and Abraxane, lipo-PTX exhibits reduced PTX leakage in the circulation, as well as diminished uptake and cardiac toxicity. A prior study demonstrated that the frequency of spontaneous calcium oscillations is significantly increased in mice several hours following
Taxol injection. Furthermore, Taxol is known to increase the expression of neuronal calcium sensor-1 in cardiomyocytes, leading to increased inositol trisphosphate receptor-dependent calcium oscillations (36). Based on the present experimental data, it was indicated that Taxol-induced arrhythmia may be directly associated with calcium concentration in cardiomyocytes. In this study, Taxol-treated mice exhibited decreased blood calcium concentrations. This finding is possibly due to the accumulation of PTX, which enhances calcium influx and induces arrhythemia in mice. In addition, calcium signaling may also be affected by modulation of ion channels, such as the sodium-potassium pump.

In previous studies, Abraxane was reported to significantly elevate antitumor activity, intratumor PTX concentration and response rate, thus resulting in decreased tumor progression, when compared with Taxol in human and mouse models $(18,55)$. However, in those studies, the PTX dose in the Abraxane-treated groups was greater than in the Taxol-treated groups. Therefore, it is unclear how Abraxane and Taxol compare, in terms of therapeutic efficacy, when the same concentration of PTX is administered. In the present study, lipo-PTX exhibited comparable antitumor effects, but limited side effects, when compared with Taxol. In conclusion, the novel formulation, lipo-PTX, diminished systemic side effects that are associated with CrEL and possessed a high therapeutic index compared with clinical PTX formulations in mouse models. Therefore, lipo-PTX may represent a feasible solution to safely increase the dose of PTX whilst reducing the limitations of CrEL-induced side effects. Based on these observations, lipo-PTX may be consider for evaluation in clinical trials to evaluate safety and efficacy in patients with breast, ovarian and liver cancer.

\section{Acknowledgements}

The authors would like to thank Dr Y.-C. Chang and Miss H.-J. Huang for assistance in Cryo-TEM imaging and also for the use of the Tecnai F20 in the Cryo-TEM Core Facility, Department of Academic Affairs and Instrument Service at Academia Sinica. In addition, the authors thank the Taiwan Animal Consortium (MOST 106-2319-B-001-004)-Taiwan Mouse Clinic, which is funded by the Ministry of Science and Technology (MOST) of Taiwan for technical support in complete blood count, blood chemistry and ECG recording. Authors also thank Y.-C. Su in the Contract Breeding and Research Division of NLAC at NARLabs for assistance in the analysis of ECG traces, and the Core Facility of the Institute of Cellular and Organismic Biology for their technical assistance.

\section{Funding}

The present study was supported by grants from Academia Sinica and Ministry of Science and Technology, Taiwan (grant nos. MOST 105-0210-01-13-01 and MOST 106-021001-15-02) to HCW.

\section{Availability of data and material}

The datasets used and/or analyzed during the current study are available from the corresponding author on reasonable request. 


\section{Authors' contributions}

HCW conceived and directed the project. STH and YPW designed and performed the experiments. STH, YPW, YHC and CTL analyzed and interpreted the data; STH and YPW wrote the manuscript. WSL and HCW designed, supervised and wrote the manuscript.

\section{Ethics approval and consent to participate}

All animal care and experimental procedures were conducted in accordance with the principles in the Guide for the Care and Use of Laboratory Animals and were approved by the Institutional Animal Care and Use Committee of Academia Sinica (Taipei, Taiwan).

\section{Patient consent for publication}

Not applicable.

\section{Competing interests}

The authors declare that they have no competing interests.

\section{References}

1. Kampan NC, Madondo MT, McNally OM, Quinn M and Plebanski M: Paclitaxel and its evolving role in the management of ovarian cancer. BioMed Res Int 2015: 413076, 2015.

2. Rowinsky EK and Donehower RC: Paclitaxel (taxol). N Engl J Med 332: 1004-1014, 1995.

3. Javeed A, Ashraf M, Riaz A, Ghafoor A, Afzal S and MukhtarMM: Paclitaxel and immune system. Eur J Pharm Sci 38: 283-290, 2009.

4. Hadzic T, Aykin-Burns N, Zhu Y, Coleman MC, Leick K, Jacobson GM and Spitz DR: Paclitaxel combined with inhibitors of glucose and hydroperoxide metabolism enhances breast cancer cell killing via $\mathrm{H}_{2} \mathrm{O}_{2}$-mediated oxidative stress. Free Radic Biol Med 48: 1024-1033, 2010.

5. Alexandre J, Hu Y, Lu W, Pelicano H and Huang P: Novel action of paclitaxel against cancer cells: Bystander effect mediated by reactive oxygen species. Cancer Res 67: 3512-3517, 2007.

6. Adams JD, Flora KP, Goldspiel BR, Wilson JW, Arbuck SG and Finley R: Taxol: A history of pharmaceutical development and current pharmaceutical concerns. J Natl Cancer Inst Monogr 15: 141-147, 1993.

7. Goldspiel BR: Clinical overview of the taxanes. Pharmacotherapy 17: 110S-125S, 1997.

8. Gelderblom H, Verweij J, Nooter K and Sparreboom A: Cremophor EL: The drawbacks and advantages of vehicle selection for drug formulation. Eur J Cancer 37: 1590-1598, 2001.

9. Szebeni J, Alving CR, Savay S, Barenholz Y, Priev A, Danino D and Talmon Y: Formation of complement-activating particles in aqueous solutions of Taxol: Possible role in hypersensitivity reactions. Int Immunopharmacol 1: 721-735, 2001.

10. U.S. Department of Health and Human Services: TAXOL ${ }^{\circledR}$ (paclitaxel) injection label. https://www.accessdata.fda.gov/ drugsatfda_docs/label/2011/020262s049lbl.pdf.

11. Weiss RB, Donehower RC, Wiernik PH, Ohnuma T, Gralla RJ Trump DL, Baker JR Jr, Van Echo DA, Von Hoff DD and Leyland-Jones B: Hypersensitivity reactions from taxol. J Clin Oncol 8: 1263-1268, 1990.

12. Sparreboom A, van Tellingen O, Nooijen WJ and Beijnen JH: Nonlinear pharmacokinetics of paclitaxel in mice results from the pharmaceutical vehicle Cremophor EL. Cancer Res 56: 2112-2115, 1996.

13. Sparreboom A, van Zuylen L, Brouwer E, Loos WJ, de Bruijn P, Gelderblom H, Pillay M, Nooter K, Stoter G and Verweij J: Cremophor EL-mediated alteration of paclitaxel distribution in human blood: Clinical pharmacokinetic implications. Cancer Res 59: 1454-1457, 1999.
14. Surapaneni MS, Das SK and Das NG: Designing Paclitaxel drug delivery systems aimed at improved patient outcomes: Current status and challenges. ISRN Pharmacol 2012: 623139, 2012.

15. Meng Z, Lv Q, Lu J, Yao H, Lv X, Jiang F, Lu A and Zhang G: Prodrug strategies for Paclitaxel. Int J Mol Sci 17: 796, 2016.

16. Yardley DA: nab-Paclitaxel mechanisms of action and delivery. J Control Release 170: 365-372, 2013.

17. Ibrahim NK, Desai N, Legha S, Soon-Shiong P, Theriault RL, Rivera E, Esmaeli B, Ring SE, Bedikian A, Hortobagyi GN, et al: Phase I and pharmacokinetic study of ABI-007, a Cremophor-free, protein-stabilized, nanoparticle formulation of paclitaxel. Clin Cancer Res 8: 1038-1044, 2002.

18. Gradishar WJ, Tjulandin S, Davidson N, Shaw H, Desai N, Bhar P, Hawkins M and O'Shaughnessy J: Phase III trial of nanoparticle albumin-bound paclitaxel compared with polyethylated castor oil-based paclitaxel in women with breast cancer. J Clin Oncol 23: 7794-7803, 2005.

19. U.S. Department of Health and Human Services: ABRAXANE ${ }^{\circledR}$ label. https://www.accessdata.fda.gov/drugsatfda_docs/ label/2015/021660s041lbl.pdf.

20. eHealthMe: Abraxane and myocardial infarction. https://www. ehealthme.com/ds/abraxane/myocardial-infarction/.

21. Feng L and Mumper RJ: A critical review of lipid-based nanoparticles for taxane delivery. Cancer Lett 334: 157-175, 2013.

22. Hong SS, Choi JY, Kim JO, Lee MK, Kim SH and Lim SJ: Development of paclitaxel-loaded liposomal nanocarrier stabilized by triglyceride incorporation. Int J Nanomedicine 11: 4465-4477, 2016 .

23. Feng Q, Yu MZ, Wang JC, Hou WJ, Gao LY, Ma XF, Pei XW, Niu YJ, Liu XY, Qiu C, et al: Synergistic inhibition of breast cancer by co-delivery of VEGF siRNA and paclitaxel via vapreotide-modified core-shell nanoparticles. Biomaterials 35: 5028-5038, 2014.

24. Immordino ML, Brusa P, Arpicco S, Stella B, Dosio F and Cattel L: Preparation, characterization, cytotoxicity and pharmacokinetics of liposomes containing docetaxel. J Control Release 91: 417-429, 2003.

25. Crosasso P, Ceruti M, Brusa P, Arpicco S, Dosio F and Cattel L: Preparation, characterization and properties of sterically stabilized paclitaxel-containing liposomes. J Control Release 63: 19-30, 2000

26. National Research Council (US) Committee for the Update of the Guide for the Care and Use of Laboratory Animals: Guide for the Care and Use of Laboratory Animals. 8th edition. The National Academies Press, Washington, DC, 2011.

27. Gregoriadis G: Drug entrapment in liposomes. FEBS Lett 36: 292-296, 1973.

28. Allen TM and Chonn A: Large unilamellar liposomes with low uptake into the reticuloendothelial system. FEBS Lett 223: 42-46, 1987.

29. Lee TY, Lin CT, Kuo SY, Chang DK and Wu HC: Peptidemediated targeting to tumor blood vessels of lung cancer for drug delivery. Cancer Res 67: 10958-10965, 2007.

30. Wu CH, Kuo YH, Hong RL and Wu HC: $\alpha$-Enolase-binding peptide enhances drug delivery efficiency and therapeutic efficacy against colorectal cancer. Sci Transl Med 7: 290ra91, 2015.

31. Bartlett GR: Phosphorus assay in column chromatography. J Biol Chem 234: 466-468, 1959.

32. Fiandaca MS, Berger MS and Bankiewicz KS: The use of convection-enhanced delivery with liposomal toxins in neurooncology. Toxins (Basel) 3: 369-397, 2011.

33. Hillaireau $\mathrm{H}$ and Couvreur P: Nanocarriers' entry into the cell: Relevance to drug delivery. Cell Mol Life Sci 66: 2873-2896, 2009.

34. Eiseman JL, Eddington ND, Leslie J, MacAuley C, Sentz DL, Zuhowski M, Kujawa JM, Young D and Egorin MJ: Plasma pharmacokinetics and tissue distribution of paclitaxel in CD2F1 mice. Cancer Chemother Pharmacol 34: 465-471, 1994.

35. Howarth FC, Calaghan SC, Boyett MR and White E: Effect of the microtubule polymerizing agent taxol on contraction, $\mathrm{Ca}^{2+}$ transient and L-type $\mathrm{Ca}^{2+}$ current in rat ventricular myocytes. J Physiol 516: 409-419, 1999.

36. Zhang K, Heidrich FM, DeGray B, Boehmerle W and Ehrlich BE: Paclitaxel accelerates spontaneous calcium oscillations in cardiomyocytes by interacting with NCS-1 and the InsP3R. J Mol Cell Cardiol 49: 829-835, 2010. 
37. Boehmerle W, Splittgerber U, Lazarus MB, McKenzie KM, Johnston DG, Austin DJ and Ehrlich BE: Paclitaxel induces calcium oscillations via an inositol 1,4,5-trisphosphate receptor and neuronal calcium sensor 1-dependent mechanism. Proc Natl Acad Sci USA 103: 18356-18361, 2006.

38. Boehmerle W, Zhang K, Sivula M, Heidrich FM, Lee Y, Jordt SE and Ehrlich BE: Chronic exposure to paclitaxel diminishes phosphoinositide signaling by calpain-mediated neuronal calcium sensor-1 degradation. Proc Natl Acad Sci USA 104: 11103-11108, 2007.

39. Drummond DC, Meyer O, Hong K, Kirpotin DB and Papahadjopoulos D: Optimizing liposomes for delivery of chemotherapeutic agents to solid tumors. Pharmacol Rev 51: 691-743, 1999.

40. McIntosh TJ: The effect of cholesterol on the structure of phosphatidylcholine bilayers. Biochim Biophys Acta 513: 43-58, 1978

41. Deniz A, Sade A, Severcan F, Keskin D, Tezcaner A and Banerjee S: Celecoxib-loaded liposomes: Effect of cholesterol on encapsulation and in vitro release characteristics. Biosci Rep 30: 365-373, 2010

42. Sharma A, Mayhew E, Bolcsak L, Cavanaugh C, Harmon P, Janoff A and Bernacki RJ: Activity of paclitaxel liposome formulations against human ovarian tumor xenografts. Int J Cancer 71: 103-107, 1997.

43. Liu Y, Ran R, Chen J, Kuang Q, Tang J, Mei L, Zhang Q, Gao H, Zhang Z and He Q: Paclitaxel loaded liposomes decorated with a multifunctional tandem peptide for glioma targeting. Biomaterials 35: 4835-4847, 2014.

44. Li XY, Zhao Y, Sun MG, Shi JF, Ju RJ, Zhang CX, Li XT, Zhao WY, Mu LM, Zeng F, et al: Multifunctional liposomes loaded with paclitaxel and artemether for treatment of invasive brain glioma. Biomaterials 35: 5591-5604, 2014.

45. Ruttala HB and Ko YT: Liposomal co-delivery of curcumin and albumin/paclitaxel nanoparticle for enhanced synergistic antitumor efficacy. Colloids Surf B Biointerfaces 128: 419-426, 2015.

46. Barbosa MV, Monteiro LO, Carneiro G, Malagutti AR, Vilela JM, Andrade MS, Oliveira MC, Carvalho-Junior AD and Leite EA: Experimental design of a liposomal lipid system: A potential strategy for paclitaxel-based breast cancer treatment. Colloids Surf B Biointerfaces 136: 553-561, 2015.

47. Assanhou AG, Li W, Zhang L, Xue L, Kong L, Sun H, Mo R and Zhang C: Reversal of multidrug resistance by co-delivery of paclitaxel and lonidamine using a TPGS and hyaluronic acid dual-functionalized liposome for cancer treatment. Biomaterials 73: 284-295, 2015.
48. Schmitt-Sody M, Strieth S, Krasnici S, Sauer B, Schulze B, Teifel M, Michaelis U, Naujoks K and Dellian M: Neovascular targeting therapy: Paclitaxel encapsulated in cationic liposomes improves antitumoral efficacy. Clin Cancer Res 9: 2335-2341, 2003.

49. Strieth S, Eichhorn ME, Werner A, Sauer B, Teifel M, Michaelis U, Berghaus A and Dellian M: Paclitaxel encapsulated in cationic liposomes increases tumor microvessel leakiness and improves therapeutic efficacy in combination with Cisplatin. Clin Cancer Res 14: 4603-4611, 2008

50. Fetterly GJ, Grasela TH, Sherman JW, Dul JL, Grahn A, Lecomte D, Fiedler-Kelly J, Damjanov N, Fishman M, Kane MP, et al: Pharmacokinetic/pharmacodynamic modeling and simulation of neutropenia during phase I development of liposome-entrapped paclitaxel. Clin Cancer Res 14: 5856-5863, 2008.

51. Slingerland M, Guchelaar HJ, Rosing H, Scheulen ME, van Warmerdam LJ, Beijnen JH and Gelderblom H: Bioequivalence of Liposome-Entrapped Paclitaxel Easy-To-Use (LEP-ETU) formulation and paclitaxel in polyethoxylated castor oil: A randomized, two-period crossover study in patients with advanced cancer. Clin Ther 35: 1946-1954, 2013.

52. Yeh ET and Bickford CL: Cardiovascular complications of cancer therapy: Incidence, pathogenesis, diagnosis, and management. J Am Coll Cardiol 53: 2231-2247, 2009.

53. Gardner ER, Dahut W and Figg WD: Quantitative determination of total and unbound paclitaxel in human plasma following Abraxane treatment. J Chromatogr B Analyt Technol Biomed Life Sci 862: 213-218, 2008

54. Sparreboom A, Scripture CD, Trieu V, Williams PJ, De T, Yang A, Beals B, Figg WD, Hawkins M and Desai N: Comparative preclinical and clinical pharmacokinetics of a cremophor-free, nanoparticle albumin-bound paclitaxel (ABI-007) and paclitaxel formulated in Cremophor (Taxol). Clin Cancer Res 11: 4136-4143, 2005.

55. Desai N, Trieu V, Yao Z, Louie L, Ci S, Yang A, Tao C, De T, Beals B, Dykes D, et al: Increased antitumor activity, intratumor paclitaxel concentrations, and endothelial cell transport of cremophor-free, albumin-bound paclitaxel, ABI-007, compared with cremophor-based paclitaxel. Clin Cancer Res 12: 1317-1324, 2006. International (CC BY-NC-ND 4.0) License. 